\title{
On a subclass of $\beta$-uniformly convex functions defined by Dziok-Srivastava linear operator
}

\author{
Jamal M. Shenan* \\ Department of Mathematics, Alazhar University - Gaza, P.O. Box. 1277, Gaza, Palestine \\ *To whom correspondence should be addressed. E-mail: shenagm@yahoo.com
}

Received 20 August 2007

http://dx.doi.org/10.11113/mjfas.v4n1.32

\section{ABSTRACT}

In this paper a new subclass of uniformly convex functions with negative coefficients defined by Dziok-Srivastava Linear operator is introduced. Characterization properties exhibited by certain fractional derivative operators of functions and the result of modified Hadmard product are discussed for this class. Further class preserving integral operator, extreme points and other interesting properties for this class are also indicated.

2000mathematics Subj. Classification: 30C45, 26 A33.

| Dziok-Srivastava Linear operator | Hadamard product |

\section{Introduction and Definitions}

Let $S$ denote the class of functions of the form

$$
f(z)=z+\sum_{n=2}^{\infty} a_{n} z^{n}
$$

which are analytic and univalent in the unit disk $U=\{z:|z|<1\}$. Also denote by $T$ the class of functions of the form

$$
f(z)=z-\sum_{n=2}^{\infty} a_{n} z^{n} \quad(z \in U) \quad\left(a_{n} \geq 0\right),
$$

which are analytic and univalent in $U$.

For functions

$$
f_{j}(z)=z-\sum_{n=2}^{\infty} a_{n, j} z^{n},\left(a_{n, j} \geq 0\right),(j=1,2)
$$


in the class $T$, the modified Hadamard product $f_{1} * f_{2}(z)$ of $f_{1}(z)$ and $f_{2}(z)$ is defined by

$$
\left(f_{1} * f_{2}\right)(z)=z-\sum_{n=2}^{\infty} a_{n, 1} a_{n, 2} z^{n} .
$$

A function $f(z) \in S$ is said to be $\beta$-uniformly starlike functions of order $\alpha$ denoted by $\beta-S(\alpha)$ iff

$$
R e\left\{\frac{z f^{\prime}(z)}{f(z)}-\alpha\right\} \geq \beta\left|\frac{z f^{\prime}(z)}{f(z)}-1\right|,
$$

for some $\alpha(-1 \leq \alpha<1), \beta \geq 0$ and all $(z \in U)$.

and is said to be $\beta$-uniformly convex of order $\alpha$ denoted by $\beta-K(\alpha)$ iff

$$
R e\left\{1+\frac{z f^{\prime \prime}(z)}{f^{\prime}(z)}-\alpha\right\} \geq \beta\left|\frac{z f^{\prime \prime}(z)}{f^{\prime}(z)}\right|,
$$

for some $\alpha(-1 \leq \alpha<1), \beta \geq 0$ and all $(z \in U)$.

The class $0-S(\alpha)=S(\alpha)$, and $0-K(\alpha)=K(\alpha)$, where $S(\alpha)$ and $K(\alpha)$ are respectively the well-known classes of starlike and convex functions of order $\alpha(0 \leq \alpha<1)$.

The classes $S(\alpha)$ and $K(\alpha)$ were first studied by Reborston [10], Schild [1], Silverman [7], and others. While the classes $\beta-S(\alpha)$ and $\beta-K(\alpha)$ were introduced and studied by Goodman [2], Rønneing [5], and Minda and Ma [4]. let

(1.7) $S^{*}(\alpha)=S(\alpha) \bigcap T, K^{*}(\alpha)=K(\alpha) \bigcap T, \beta-S^{*}(\alpha)=[\beta-S(\alpha)] \bigcap T$, and $\quad \beta-K^{*}(\alpha)=[\beta-K(\alpha)] \bigcap T$.

For $\alpha_{i} \in C(i=1,2,3 \ldots, l)$ and $\beta_{j} \in C-\{0,-1,-2, .\}.(j=1,2,3, \ldots, m)$, the generalized hypergeometric function is defined by

$$
\begin{gathered}
{ }_{l} F_{m}\left(\alpha_{1}, \ldots, \alpha_{l} ; \beta_{1}, \ldots, \beta_{m}\right)=\sum_{n=0}^{\infty} \frac{\left(\alpha_{1}\right)_{n} \ldots\left(\alpha_{l}\right)_{n}}{\left(\beta_{1}\right)_{n} \ldots\left(\beta_{m}\right)_{n}} \cdot \frac{z^{n}}{n !}, \\
\left(l \leq m+1 ; m \in N_{0}=\{0,1,2 \ldots\}\right),
\end{gathered}
$$

where $(a)_{n}$ is the Pochhammer symbol defined by

$$
(a)_{n}=\frac{\Gamma(a+n)}{\Gamma(a)}= \begin{cases}1 ; & \mathrm{n}=0 \\ a(a+1)(a+2) \ldots(a+n+1), & n \in N=1,2, \ldots\end{cases}
$$


Corresponding to the function $h\left(\alpha_{1}, \ldots, \alpha_{l} ; \beta_{1}, \ldots, \beta_{m} ; z\right)=z_{l} F_{m}\left(\alpha_{1}, \ldots, \alpha_{l} ; \beta_{1}, \ldots, \beta_{m}\right)$ the Dziok-Srivastava operator [9], $H_{m}^{L}\left(\alpha_{1}, \ldots, \alpha_{l} ; \beta_{1}, \ldots, \beta_{m}\right)$ is defined by

$$
\begin{aligned}
H_{m}^{L}\left(\alpha_{1}, \ldots, \alpha_{l} ; \beta_{1}, \ldots, \beta_{m}\right) f(z) & =h\left(\alpha_{1}, \ldots, \alpha_{l} ; \beta_{1}, \ldots, \beta_{m} ; z\right) * f(z) \\
= & z+\sum_{n=2}^{\infty} \frac{\left(\alpha_{1}\right)_{n-1} \ldots\left(\alpha_{l}\right)_{n-1}}{\left(\beta_{1}\right)_{n-1} \ldots\left(\beta_{m}\right)_{n-1}} a_{n} \frac{z^{n}}{(n-1) !} .
\end{aligned}
$$

It is well known [9] that

(1.11) $\alpha_{1} H_{m}^{L}\left(\alpha_{1}+1, \ldots, \alpha_{l} ; \beta_{1}, \ldots, \beta_{m}\right) f(z)=z\left[H_{m}^{L}\left(\alpha_{1}, \ldots, \alpha_{l} ; \beta_{1}, \ldots, \beta_{m}\right) f(z)\right]^{\prime}$

To make the notation simple, we write,

$$
+\left(\alpha_{1}-1\right) H_{m}^{L}\left(\alpha_{1}, \ldots, \alpha_{l} ; \beta_{1}, \ldots, \beta_{m}\right) f(z) .
$$

$$
H_{m}^{L}\left[\alpha_{1}\right] f(z)=H_{m}^{L}\left(\alpha_{1}, \ldots, \alpha_{l} ; \beta_{1}, \ldots, \beta_{m}\right) f(z) .
$$

We note that special cases of the Dziok-Srivastava operator $H_{m}^{L}\left[\alpha_{1}\right]$ include the Hohlov linear operator [15], the Carlson-Shafer operator[3], the Ruschweyh derivative operator[13], the Srivastava-Owa fractional operators [14], and many others.

Now using $H_{m}^{L}\left[\alpha_{1}\right]$ we define the following subclass of analytic function.

Definition 1. For $-1 \leq \alpha<1, \beta \geq 0, \alpha_{i} \in C(i=1,2,3 \ldots, l)$ and $\beta_{j} \in C-\{0,-1,-2, .\}.(j=1,2,3, \ldots, m)$, we let $S_{m}^{l}\left(\alpha_{l}, \beta_{m}, \beta, \alpha\right)$ be the subclass of $\mathrm{S}$ consisting of functions $f(z)$ of the form (1.1) and satisfying the following condition

$$
\operatorname{Re}\left\{\alpha_{1} \frac{H_{m}^{L}\left[\alpha_{1}+1\right] f(z)}{H_{m}^{L}\left[\alpha_{1}\right] f(z)}+1-\alpha_{1}-\alpha\right\}>\beta\left|\alpha_{1} \frac{H_{m}^{L}\left[\alpha_{1}+1\right] f(z)}{H_{m}^{L}\left[\alpha_{1}\right] f(z)}-\alpha_{1}\right|, z \in U
$$

also let $T_{m}^{l}\left(\alpha_{l}, \beta_{m}, \beta, \alpha\right)=S_{m}^{l}\left(\alpha_{l}, \beta_{m}, \beta, \alpha\right) \bigcap T$.

It may be noted that the class $T_{m}^{l}\left(\alpha_{l}, \beta_{m}, \beta, \alpha\right)$ extends the classes of starlike, convex, $\beta$-uniformly starlike and $\beta$-uniformly convex for suitable choice of $l, m, \alpha_{i}, \beta_{j}, \beta$ and $\alpha$. For example

i) For $l=1, m=0, \alpha_{1}=1$ the class $T_{m}^{l}\left(\alpha_{l}, \beta_{m}, \beta, \alpha\right)$ reduces to the class of $\beta$-uniformly starlike functions.

(ii) For $l=1, m=0, \alpha_{1}=2$ we obtain the class of $\beta$-uniformly convex function. 
(iii) For $l=3, m=2, \alpha_{1}=2-\gamma+\eta, \alpha_{2}=2, \alpha_{3}=1, \beta_{1}=2-\gamma, \beta_{2}=$ $2-\mu+\eta, \beta=1$ we obtain the class studied in [6].

Several other classes studied by various research workers can be obtained from the class $T_{m}^{l}\left(\alpha_{l}, \beta_{m}, \beta, \alpha\right)$.

Following Raina and Nahar [12], the fractional derivative operator $D_{0, z}^{\lambda, \mu, \eta}$ of a function $f(z)$ is defined as follows.

Definition 2. For $m-1 \leq \lambda<m ; m \in N$ and $\mu, \eta \in R$

$$
\begin{aligned}
D_{0, z}^{\lambda, \mu, \eta} f(z) & =\frac{d^{m}}{d z^{m}}\left\{\frac{z^{\lambda-\mu}}{\Gamma(m-\lambda)} \int_{0}^{z}(z-t)^{m-\lambda-1}\right. \\
& \left.{ }_{2} F_{1}\left(\mu-\lambda, m-\eta ; m-\lambda ; 1-\frac{t}{z}\right) f(t) d t\right\},
\end{aligned}
$$

where the function $f(z)$ is analytic in a simply connected region of the $\mathrm{z}$ plane containing the region, with the order

$$
f(z)=o\left(|z|^{r}\right), z \longrightarrow 0
$$

where $r>\max \{0, \mu-\eta\}-1$ and the multiplicity of $(z-t)^{m-\lambda-1}$ is removed by requiring $\log (z-t)$ to be real when $(z-t)>0$ and is well defined in the unit disk.

The operator defined by (1.13) includes the well known Riemann-Liouville fractional derivative operator ${ }_{0} D_{z}^{\lambda} f(z)[11]$. Indeed we have

$$
D_{0, z}^{\lambda, \lambda, \eta} f(z)={ }_{0} D_{z}^{\lambda} f(z)
$$

The fractional operator $J_{0 . z}^{\lambda, \mu, \eta}$ is defined in terms of $D_{0, z}^{\lambda, \mu, \eta}$ as follows.

$$
\begin{aligned}
& J_{0, z}^{\lambda, \mu, \eta} f(z)=\frac{\Gamma(2-\mu) \Gamma(2-\lambda+\eta)}{\Gamma(2-\mu+\eta)} z^{\mu} D_{0, z}^{\lambda, \mu, \eta} f(z), \\
& (\lambda \geq 0 ; \mu<2 ; \eta>\max \{\lambda, \mu\}-2)
\end{aligned}
$$

Lemma 1. [12]. if $\lambda \geq 0 ; n>\max \{0, \mu-\eta\}-1$, then

$$
D_{0, z}^{\lambda, \mu, \eta} z^{n}=\frac{\Gamma(n+1) \Gamma(n-\mu+\eta+1)}{\Gamma(n-\mu+1) \Gamma(n-\lambda+\eta+1)} z^{n-\mu} .
$$


Applying Lemma 1 for the function $f(z)$ defined by (1.1) we have from (1.15)

$$
J_{0, z}^{\lambda, \mu, \eta} f(z)=z+\sum_{n=2}^{\infty} \frac{(2)_{n-1}(2+\eta-\mu)_{n-1}}{(2-\mu)_{n-1}(2+\eta-\lambda)_{n-1}} a_{n} z^{n}, \quad(n \geq 2)
$$

\section{Coefficient Estimates.}

Theorem 1. A function $f(z)$ defined by (1.2) is in the class $T_{m}^{l}\left(\alpha_{l}, \beta_{m}, \beta, \alpha\right)$, $-1 \leq \alpha<1, \beta \geq 0, \alpha_{i} \in C(i=1,2,3 \ldots, l)$ and $\beta_{j} \in C-\{0,-1,-2, .$. $(j=1,2,3, \ldots, m)$, if and only if

$$
\sum_{n=2}^{\infty}\{n(1+\beta)-(\alpha+\beta)\} \frac{\phi(n)}{(n-1) !} a_{n} \leq(1-\alpha)
$$

where

$$
\phi(n)=\frac{\left(\alpha_{1}\right)_{n-1} \ldots\left(\alpha_{l}\right)_{n-1}}{\left(\beta_{1}\right)_{n-1} \ldots\left(\beta_{m}\right)_{n-1}} .
$$

and the result is sharp.

Proof. Let $f(z) \in T_{m}^{l}\left(\alpha_{l}, \beta_{m}, \beta, \alpha\right)$ and $z$ be real then by virtue of (1.10) and (1.11) we have

$$
\frac{1-\sum_{n=2}^{\infty} n \frac{\phi(n)}{(n-1) !} a_{n} z^{n-1}}{1-\sum_{n=2}^{\infty} \frac{\phi(n)}{(n-1) !} a_{n} z^{n-1}}-\alpha \geq \beta\left|\frac{\sum_{n=2}^{\infty}(n-1) \frac{\phi(n)}{(n-1) !} a_{n} z^{n-1}}{1-\sum_{n=2}^{\infty} \frac{\phi(n)}{(n-1) !} a_{n} z^{n-1}}\right| .
$$

Letting $z \rightarrow 1$ along the real axis, we obtain the desire inequality (2.1).

Conversely, assuming that (2.1) holds, then we show that

$$
\beta\left|\alpha_{1} \frac{H_{m}^{L}\left[\alpha_{1}+1\right] f(z)}{H_{m}^{L}\left[\alpha_{1}\right] f(z)}-\alpha_{1}\right|-\operatorname{Re}\left\{\alpha_{1} \frac{H_{m}^{L}\left[\alpha_{1}+1\right] f(z)}{H_{m}^{L}\left[\alpha_{1}\right] f(z)}-\alpha_{1}\right\} \leq 1-\alpha
$$

We have

$$
\begin{aligned}
& \beta\left|\alpha_{1} \frac{H_{m}^{L}\left[\alpha_{1}+1\right] f(z)}{H_{m}^{L}\left[\alpha_{1}\right] f(z)}-\alpha_{1}\right|-\operatorname{Re}\left\{\alpha_{1} \frac{H_{m}^{L}\left[\alpha_{1}+1\right] f(z)}{H_{m}^{L}\left[\alpha_{1}\right] f(z)}-\alpha_{1}\right\} \\
& \leq(1+\beta)\left|\alpha_{1} \frac{H_{m}^{L}\left[\alpha_{1}+1\right] f(z)}{H_{m}^{L}\left[\alpha_{1}\right] f(z)}-\alpha_{1}\right|
\end{aligned}
$$




$$
\leq \frac{(1+\beta) \sum_{n=2}^{\infty}(n-1) \frac{\phi(n)}{(n-1) !} a_{n}}{1-\sum_{n=2}^{\infty} \frac{\phi(n)}{(n-1) !} a_{n}} .
$$

This expression is bounded above by $(1-\alpha)$ if

$$
\sum_{n=2}^{\infty}\{n(1+\beta)-(\alpha+\beta)\} \frac{\phi(n)}{(n-1) !} a_{n} \leq(1-\alpha)
$$

The equality in (2.1) is attained for the function

$$
f(z)=z-\frac{(1-\alpha)(n-1) !}{\{n(1+\beta)-(\alpha+\beta)\} \phi(n)} z^{n} .
$$

and hence the proof is complete.

Corollary 1. Let the function $f(z)$ defined by (1.2) be in the class $T_{m}^{l}\left(\alpha_{l}, \beta_{m}, \beta, \alpha\right)$, $-1 \leq \alpha<1, \beta \geq 0, \alpha_{i} \in C(i=1,2,3 \ldots, l)$ and $\beta_{j} \in C-\{0,-1,-2, .$. $(j=1,2,3, \ldots, m)$.Then

$$
a_{n} \leq \frac{(1-\alpha)(n-1) !}{\{n(1+\beta)-(\alpha+\beta)\} \phi(n)}, \quad n \geq 2
$$

\section{Characterization Properties.}

Theorem 2. Let $a_{i}>0(i=1,2,3 \ldots, p)$ and $b_{j}>0(j=1,2,3, \ldots, q)$ such that $\prod_{j=1}^{q} b_{j} \geq \prod_{i=1}^{p} a_{i}$. Also let the function $f(z)$ defined by (1.2) satisfy

$$
\sum_{n=2}^{\infty} \frac{\{n(1+\beta)-(\alpha+\beta)\}}{1-\alpha} \frac{\phi(n)}{(n-1) !} a_{n} \leq \frac{\prod_{j=1}^{q} b_{j}}{\prod_{i=1}^{p} a_{i}}
$$

for $-1 \leq \alpha<1, \beta \geq 0$. then

$$
H_{q}^{p}\left[a_{1}\right] f(z) \in T_{m}^{l}\left(\alpha_{l}, \beta_{m}, \beta, \alpha\right),
$$

where $\phi(n)$ is given by $(2.2)$. 
Proof. We have from (1.10)

$$
H_{q}^{p}\left[a_{1}\right] f(z)=H_{q}^{p}\left(a_{1}, \ldots, a_{p} ; b_{1}, \ldots, b_{q}\right) f(z)=z-\sum_{n=2}^{\infty} \delta(n) a_{n} z^{n}
$$

where

$$
\delta(n)=\frac{\left(a_{1}\right)_{n-1} \ldots\left(a_{p}\right)_{n-1}}{\left(b_{1}\right)_{n-1} \ldots\left(b_{q}\right)_{n-1}(n-1) !}(n \geq 2) .
$$

Under the conditions stated in the theorem, we observe that the function $\delta(n)$ is non-increasing, that is, it satisfies the inequality $\delta(n+1) \leq \delta(n)$ for all $n \geq 2$, and thus we have

$$
0<\delta(n) \leq \delta(2)=\frac{\prod_{i=1}^{p} a_{i}}{\prod_{j=1}^{q} b_{j}}
$$

Therefore, (3.1) and (3.4) yield

$$
\begin{aligned}
\sum_{n=2}^{\infty} \frac{\{n(1+\beta)-(\alpha+\beta)\}}{(1-\alpha)} & \frac{\phi(n)}{(n-1) !} a_{n} \delta(n) \\
& \leq \delta(2) \sum_{n=2}^{\infty} \frac{\{n(1+\beta)-(\alpha+\beta)\}}{(1-\alpha)} \frac{\phi(n)}{(n-1) !} \\
& \leq 1
\end{aligned}
$$

Hence by Theorem 1, we conclude that

$$
H_{q}^{p}\left[a_{1}\right] f(z) \in T_{m}^{l}\left(\alpha_{l}, \beta_{m}, \beta, \alpha\right) .
$$

Remark 1. The equality in (3.1) is attained for the function $f(z)$ defined by

$$
f(z)=z-\frac{(1-\alpha)\left(\prod_{j=1}^{m} \beta_{j}\right)\left(\prod_{j=1}^{q} b_{j}\right)}{(2+\beta-\alpha)\left(\prod_{i=1}^{l} \alpha_{i}\right)\left(\prod_{i=1}^{p} a_{i}\right)} z^{2}
$$


Corollary 2. Let $\lambda, \mu, \eta \in \Re$ such that

$$
\lambda \geq 0, \mu<2, \max (\lambda, \mu)-2<\eta \leq \frac{\lambda(\mu-3)}{\mu}
$$

also, let the function $f(z)$ defined by (1.2) satisfy

$$
\sum_{n=2}^{\infty} \frac{\{n(1+\beta)-(\alpha+\beta)\}}{1-\alpha} a_{n} \leq \frac{(2-\mu)(2+\eta-\lambda)}{2(2+\eta-\mu)}
$$

for $-1 \leq \alpha<1, \beta \geq 0$. then

$$
J_{0, z}^{\lambda, \mu, \eta} f(z) \in \beta-S^{*}(\alpha)
$$

Proof. The corollary follows from Theorem 2 by setting $p=3, q=2, a_{1}=$ $1, a_{2}=2, a_{3}=2+\eta-\mu, b_{1}=2-\mu, b_{2}=2+\eta-\lambda$ and $l=1, m=0, \alpha_{1}=1$.

Remark 2. In Corollary 2, if the function $f(z)$ is given by (1.1) and $\beta=0$, we obtain the corresponding result due to Raina and Nahar [12, P. 4, Theorem 1].

Corollary 3. Under the conditions stated in (3.7), let the function $f(z)$ defined by (1.2) satisfy

$$
\sum_{n=2}^{\infty} \frac{n\{n(1+\beta)-(\alpha+\beta)\}}{1-\alpha} a_{n} \leq \frac{(2-\mu)(2+\eta-\lambda)}{2(2+\eta-\mu)}
$$

for $-1 \leq \alpha<1, \beta \geq 0$. then

$$
J_{0, z}^{\lambda, \mu, \eta} f(z) \in \beta-k^{*}(\alpha)
$$

Proof. The corollary follows from Theorem 2 by setting $p=3, q=2, a_{1}=$ $1, a_{2}=2, a_{3}=2+\eta-\mu, b_{1}=2-\mu, b_{2}=2+\eta-\lambda$ and $l=1, m=0, \alpha_{1}=2$.

Remark 3. In Corollary 3, if the function $f(z)$ is given and (1.1), and $\beta=0$, we obtain the corresponding result due to Raina and Nahar $[12$, p. 5 , Theorem 2 ]. 


\section{Results Involving Modified Hadamard Product.}

Theorem 3. Let $\alpha_{i}>0(i=1,2,3 \ldots, l)$ and $\beta_{j}>0(j=1,2,3, \ldots, m)$ such that $\prod_{j=1}^{m} \beta_{j} \geq \prod_{i=1}^{l} \alpha_{i}$. Also for functions $f_{j}(z)(j=1,2)$ defined by (1.3), let $f_{1}(z) \in T_{m}^{l}\left(\alpha_{l}, \beta_{m}, \beta, \alpha\right)$ and $f_{2}(z) \in T_{m}^{l}\left(\alpha_{l}, \beta_{m}, \beta, \gamma\right)$.

then $f_{1} * f_{2}(z) \in T_{m}^{l}\left(\alpha_{l}, \beta_{m}, \beta, \delta\right)$ where

$$
\delta=1-\frac{(1+\beta)(1-\alpha)(1-\gamma)\left(\prod_{j=1}^{m} \beta_{j}\right)}{(2+\beta-\alpha)(2+\beta-\gamma)\left(\prod_{i=1}^{l} \alpha_{i}\right)-(1-\alpha)(1-\gamma)\left(\prod_{j=1}^{m} \beta_{j}\right)} .
$$

and the result is sharp.

Proof. To prove the theorem it is sufficient to assert that

$$
\sum_{n=2}^{\infty} \frac{\{n(1+\beta)-(\delta+\beta)\}}{1-\delta} \frac{\phi(n)}{(n-1) !} a_{n, 1} a_{n, 2} \leq 1,
$$

where $\phi(n)$ is defined in (2.2) and $\delta$ is defined in (4.1). Now by virtue of Cauchy-Schwarz inequality and Theorem 1, it follows that

$$
\sum_{n=2}^{\infty} \frac{\{n(1+\beta)-(\alpha+\beta)\}^{1 / 2}\{n(1+\beta)-(\gamma+\beta)\}^{1 / 2}}{\sqrt{(1-\alpha)(1-\gamma)}} \frac{\phi(n)}{(n-1) !} \sqrt{a_{n, 1} a_{n, 2}} \leq 1
$$

Hence (4.2) is true if

$$
\begin{aligned}
& \frac{\{n(1+\beta)-(\delta+\beta)\}}{1-\delta} \frac{\phi(n)}{(n-1) !} a_{n, 1} a_{n, 2} \\
& \quad \leq \frac{\{n(1+\beta)-(\alpha+\beta)\}^{1 / 2}\{n(1+\beta)-(\gamma+\beta)\}^{1 / 2}}{\sqrt{(1-\alpha)(1-\gamma)}} \frac{\phi(n)}{(n-1) !} \sqrt{a_{n, 1} a_{n, 2}} \\
& \quad \leq 1
\end{aligned}
$$

or, equivalently

$$
\begin{aligned}
\sqrt{a_{n, 1} a_{n, 2}} \leq \frac{\{n(1+\beta)-(\alpha+\beta)\}^{1 / 2}\{n(1+\beta)-(\gamma+\beta)\}^{1 / 2}}{\sqrt{(1-\alpha)(1-\gamma)}} & \\
& \times \frac{1-\delta}{\{n(1+\beta)-(\delta+\beta)\}}
\end{aligned}
$$


By virtue of (4.3), (4.2) is true if

$$
\begin{aligned}
& \frac{\sqrt{(1-\alpha)(1-\gamma)}(n-1) !}{\{n(1+\beta)-(\alpha+\beta)\}^{1 / 2}\{n(1+\beta)-(\gamma+\beta)\}^{1 / 2} \phi(n)} \\
& \leq \frac{\{n(1+\beta)-(\alpha+\beta)\}^{1 / 2}\{n(1+\beta)-(\gamma+\beta)\}^{1 / 2}}{\sqrt{(1-\alpha)(1-\gamma)}} \\
& \times \frac{1-\delta}{\{n(1+\beta)-(\delta+\beta)\}}
\end{aligned}
$$

which yields

$$
\delta \leq 1-\frac{(n-1)(\beta+1)(1-\alpha)(1-\gamma)(n-1) !}{\{n(1+\beta)-(\alpha+\beta)\}\{n(1+\beta)-(\gamma+\beta)\} \phi(n)-(1-\alpha)(1-\gamma)(n-1) !}
$$

Under the stated conditions in the theorem, we observe that the function $\phi(n)$ is a decreasing for $n(n \geq 2)$, and thus (4.5) is satisfied if $\delta$ is given by (4.1). Finally the result is sharp for

$$
\begin{aligned}
& f_{1}(z)=z-\frac{(1-\alpha)\left(\prod_{j=1}^{m} \beta_{j}\right)}{(2+\beta-\alpha)\left(\prod_{i=1}^{l} \alpha_{i}\right)} z^{2} \\
& f_{2}(z)=z-\frac{(1-\gamma)\left(\prod_{j=1}^{m} \beta_{j}\right)}{(2+\beta-\gamma)\left(\prod_{i=1}^{l} \alpha_{i}\right)} z^{2}
\end{aligned}
$$

Theorem 4. Under the conditions stated in Theorem 3, let the functions $f_{j}(z)(j=1,2)$ defined by $(1.3)$, be in the class $T_{m}^{l}\left(\alpha_{l}, \beta_{m}, \beta, \alpha\right)$. Then $f_{1} * f_{2}(z) \in T_{m}^{l}\left(\alpha_{l}, \beta_{m}, \beta, \delta\right)$ where

$$
\delta=1-\frac{(1+\beta)(1-\alpha)^{2}\left(\prod_{j=1}^{m} \beta_{j}\right)}{(2+\beta-\alpha)^{2}\left(\prod_{i=1}^{l} \alpha_{i}\right)-(1-\alpha)^{2}\left(\prod_{j=1}^{m} \beta_{j}\right)} .
$$

Proof. The result follows by setting $\alpha=\gamma$ in Theorem 3 . 
Theorem 5. Under the conditions stated in Theorem 3, let the functions $f_{j}(z)(j=1,2)$ defined by $(1.3)$, be in the class $T_{m}^{l}\left(\alpha_{l}, \beta_{m}, \beta, \alpha\right)$. Then

$$
h(z)=z-\sum_{n=2}^{\infty}\left(a_{n, 1}^{2}+a_{n, 2}^{2}\right) z^{2}
$$

is in the class $T_{m}^{l}\left(\alpha_{l}, \beta_{m}, \beta, \delta\right)$ where

$$
\delta=1-\frac{2(1+\beta)(1-\alpha)^{2}\left(\prod_{j=1}^{m} \beta_{j}\right)}{(2+\beta-\alpha)^{2}\left(\prod_{i=1}^{l} \alpha_{i}\right)-2(1-\alpha)^{2}\left(\prod_{j=1}^{m} \beta_{j}\right)} .
$$

Proof. In view of Theorem 1, it is sufficient to prove that

$$
\sum_{n=2}^{\infty} \frac{\{n(1+\beta)-(\delta+\beta)\}}{1-\delta} \frac{\phi(n)}{(n-1) !}\left(a_{n, 1}^{2}+a_{n, 2}^{2}\right) \leq 1,
$$

where $\phi(n)$ is defined in (2.2) and $\delta$ is defined in (4.8).

as $f_{j}(z) \in T_{m}^{l}\left(\alpha_{l}, \beta_{m}, \beta, \alpha\right)(j=1,2)$, Theorem 1 yields

$$
\begin{gathered}
\sum_{n=2}^{\infty}\left[\frac{\{n(1+\beta)-(\alpha+\beta)\} \phi(n)}{(1-\alpha)(n-1) !}\right]^{2} a_{n, j}^{2} \\
\leq \sum_{n=2}^{\infty}\left[\frac{\{n(1+\beta)-(\alpha+\beta)\} \phi(n)}{(1-\alpha)(n-1) !} a_{n, j}\right]^{2} \\
\leq 1
\end{gathered}
$$

hence

$$
\sum_{n=2}^{\infty} \frac{1}{2}\left[\frac{\{n(1+\beta)-(\alpha+\beta)\} \phi(n)}{(1-\alpha)(n-1) !}\right]^{2}\left(a_{n, 1}^{2}+a_{n, 2}^{2}\right) \leq 1
$$

(4.9) is true if

$$
\begin{aligned}
\frac{\{n(1+\beta)-(\delta+\beta)\}}{1-\delta} & \frac{\phi(n)}{(n-1) !}\left(a_{n, 1}^{2}+a_{n, 2}^{2}\right) \\
\leq & \frac{1}{2}\left[\frac{\{n(1+\beta)-(\alpha+\beta)\} \phi(n)}{(1-\alpha)(n-1) !}\right]^{2}\left(a_{n, 1}^{2}+a_{n, 2}^{2}\right),
\end{aligned}
$$


that is, if

$$
\delta \leq 1-\frac{2(n-1)(1+\beta)(1-\alpha)^{2}(n-1) !}{[n(1+\beta)-(\alpha+\beta)]^{2} \phi(n)-2(1-\alpha)^{2}(n-1) !} .
$$

Under the stated conditions in the theorem, we observe that the function $\phi(n)$ is a decreasing for $n(n \geq 2)$, and thus (4.11) is satisfied if $\delta$ is given by (4.8).

5. Extreme points of the class $T_{m}^{l}\left(\alpha_{l}, \beta_{m}, \beta, \alpha\right)$.

Theorem 6. Let $f_{1}(z)=z$ and

$$
f_{n}(z)=z-\frac{(1-\alpha)(n-1) !}{\{n(1+\beta)-(\alpha+\beta)\} \phi(n)} z^{n}, \quad(n \geq 2) .
$$

Then $f(z) \in T_{m}^{l}\left(\alpha_{l}, \beta_{m}, \beta, \alpha\right)$ if and only if it can be expressed in the form

$$
f(z)=\lambda_{1} f_{1}(z)+\sum_{n=2}^{\infty} \lambda_{n} f_{n}(z),
$$

where $\lambda_{n} \geq 0$ and $\sum_{n=1}^{\infty} \lambda_{n}=1$, and $\phi(n)$ is given in (2.2).

Proof. Let (5.2) holds, then by (5.1) we have

$$
f(z)=z-\sum_{n=2}^{\infty} \frac{(1-\alpha)(n-1) !}{\{n(1+\beta)-(\alpha+\beta)\} \phi(n)} \lambda_{n} z^{n} .
$$

Now

$$
\begin{aligned}
\sum_{n=2}^{\infty}\{n(1+\beta)- & (\alpha+\beta)\} \frac{\phi(n)}{(n-1) !} a_{n} \\
= & \sum_{n=2}^{\infty}\{n(1+\beta)-(\alpha+\beta)\} \frac{\phi(n)}{(n-1) !} \\
& \times \frac{(1-\alpha)(n-1) !}{\{n(1+\beta)-(\alpha+\beta)\} \phi(n)} \lambda_{n} \\
= & (1-\alpha) \sum_{n=2}^{\infty} \lambda_{n} \\
\leq & (1-\alpha) \sum_{n=1}^{\infty} \lambda_{n} \\
\leq & 1-\alpha .
\end{aligned}
$$


Hence by Theorem 1, $f(z) \in T_{m}^{l}\left(\alpha_{l}, \beta_{m}, \beta, \alpha\right)$.

Conversely, suppose $f(z) \in T_{m}^{l}\left(\alpha_{l}, \beta_{m}, \beta, \alpha\right)$. Since

$$
a_{n} \leq \frac{(1-\alpha)(n-1) !}{\{n(1+\beta)-(\alpha+\beta)\} \phi(n)}, \quad(n \geq 2)
$$

setting $\lambda_{n}=\frac{\{n(1+\beta)-(\alpha+\beta)\} \phi(n)}{(1-\alpha)(n-1) !} a_{n}$ and $\lambda_{1}=1-\sum_{n=2}^{\infty} \lambda_{n}$, we get (5.2). This completes the proof of the theorem.

\section{Closure properties}

Theorem 7. Let the functions $f_{j}(z)$ defined by (1.3) be in the class $T_{m}^{l}\left(\alpha_{l}, \beta_{m}, \beta, \alpha\right)$. Then the function $h(z)$ defined by

$$
h(z)=z-\sum_{n=2}^{\infty} d_{n} z^{n}
$$

belongs to $T_{m}^{l}\left(\alpha_{l}, \beta_{m}, \beta, \alpha\right)$, where

$$
d_{n}=\frac{1}{m} \sum_{j=1}^{m} a_{n, j}, \quad\left(a_{n, j} \geq 0\right) .
$$

Proof. Since $f_{j}(z) \in T_{m}^{l}\left(\alpha_{l}, \beta_{m}, \beta, \alpha\right)$, it follows from Theorem 1 that

$$
\sum_{n=2}^{\infty}\{n(1+\beta)-(\alpha+\beta)\} \frac{\phi(n)}{(n-1) !} a_{n, j} \leq(1-\alpha),
$$

where $\phi(n)$ is given by (2.2). Therefore

$$
\begin{aligned}
\sum_{n=2}^{\infty}\{n(1+\beta)- & (\alpha+\beta)\} \frac{\phi(n)}{(n-1) !} d_{n} \\
& =\sum_{n=2}^{\infty}\{n(1+\beta)-(\alpha+\beta)\} \frac{\phi(n)}{(n-1) !}\left(\frac{1}{m} \sum_{j=1}^{m} a_{n j}\right) \\
\leq & 1-\alpha,
\end{aligned}
$$

by (6.1), which shows that $T_{m}^{l}\left(\alpha_{l}, \beta_{m}, \beta, \alpha\right)$. 


\section{Integral transforms}

Recently, Jung, Kim and Srivastava [8] introduced the following oneparameter family of integral operators

$$
I^{\sigma} f(z)=\frac{2^{\sigma}}{z \Gamma(\sigma)} \int_{0}^{z}\left(\log \frac{z}{t}\right)^{\sigma-1} f(t) d t, \quad(\sigma>0) .
$$

Theorem 8. Let the function $f(z)$ defined by (1.2) be the class $T_{m}^{l}\left(\alpha_{l}, \beta_{m}, \beta, \alpha\right)$. Then the integral transforms (7.1) belongs to $T_{m}^{l}\left(\alpha_{l}, \beta_{m}, \beta, \alpha\right)$.

Proof. Using (1.2) and (7.1) we get

$$
I^{\sigma} f(z)=z-\sum_{n=2}^{\infty}\left(\frac{2}{1+n}\right)^{\sigma} a_{n} z^{n} .
$$

Therefore,

$$
\begin{aligned}
\sum_{n=2}^{\infty}\{n(1+\beta)-(\alpha & +\beta)\} \frac{\phi(n)}{(n-1) !}\left(\frac{2}{1+n}\right)^{\sigma} a_{n} \\
& \leq \sum_{n=2}^{\infty}\{n(1+\beta)-(\alpha+\beta)\} \frac{\phi(n)}{(n-1) !} a_{n} \\
& \leq 1-\alpha .
\end{aligned}
$$

which implies that $T_{m}^{l}\left(\alpha_{l}, \beta_{m}, \beta, \alpha\right)$.

\section{References}

[1] A. Schild, On starlike functions of order $\alpha$, Amer. J. Math. 87(1965), 65-70.

[2] A. W. Goodman, On uniformly convex functions, Ann. Polon. Math. 56(1991),87-92.

[3] B. C.Carlson and D. B. Shaffer,Starlike and pre-starlike hypergeometric functions, SIAM J. Math. Anal. 15(1984),737-745.

[4] D. Minda and W. Ma, Uniformly convex functions, Ann. Polon. Math. 57(1992),165-175. 
[5] F. Rønning, Uniformly convex functions and a corresponding class of starlike functions, Proc. Amer. Math. Soc. 118(1993),189-196.

[6] G. Mur., T. Rosy and M. Darus, A subclass of uniformly convex functions associated with certain fractional calculus operators, J. Ineq. Pure and Appl. Math.,6(3) Art.,86(2005), 1-10.

[7] H. Silverman, Univalent functions with negative coefficients, Proc Amer. Math. Soc. 51(1975),109-116.

[8] I. B. Jung, Y. C. Kim and H. M. Srivastava, The Hardly space of analytic functions associated with certain one-parameter families of integral transform, J. Math. Anal. Appl., 179(1993), 138-147.

[9] J. Dziok and H. M. Srivastava, Certain subclass f analytic functions associated with the generalized hypergeometric function, Integral Transform Spec. funct., 14(2003), 7-18.

[10] M. S. Robertson, On the theory of univalent functions, Ann. of Math. 37(1936),374-408.

[11] R. K. Raina and M. Saigo, A note on fractional calculus operators involving Fox'H-function on space $F_{p, \mu}$, Recent Advances in Fractional Calculus, Global Publishing Co. Sauk Rapids, 1993, pp. 219-229.

[12] R. K. Raina and T. S. Nahar, Characterization properties for starlikeness and convexity of some subclasses of analytic functions involving a class of fractional derivative operator. Acta Math. Univ. Comenianae. Vol.LXIX,1(2000),1-8.

[13] St. Ruscheweyh, New criteria for univalent functions, Proc. Amer. Math. Soc., 49(1975), 109-115.

[14] S. Owa and H. M. Srivastava, Univalent and starlike generalized hypergeometric functions, Canad. J. Math. 39 (5)(1987), 1057-1077.

[15] Yu. E. Hohlov, Operators and operations in the class of univalent functions, Izv. Vyss. Ucebn. Zaved. Mat., 10(1978), 83-89. 